\title{
Comunicação
}

[Communication]

\section{Hepatozoonose canina: achados clínico-epidemiológicos em três casos}

[Canine hepatozoonosis: clinic-epidemiological data in three cases]

\author{
D.M. Aguiar ${ }^{1,3}$, M.G. Ribeiro ${ }^{2}$, W.B. Silva ${ }^{2}$, J.G. Dias $J r^{2}$, J. Megid ${ }^{2}$, A.C. Paes ${ }^{2}$ \\ ${ }^{1}$ Depto. Medicina Veterinária Preventiva e Saúde Animal - FMVZ - USP \\ Av. Prof. Dr. Orlando Marques de Paiva, 87 \\ 05508-000 - São Paulo, SP \\ ${ }^{2}$ Depto. Higiene Veterinária e Saúde Pública - FMVZ-UNESP-Botucatu, SP \\ ${ }^{3}$ Bolsista do $\mathrm{CNPq}$
}

A hepatozoonose é uma doença descrita em vários países, causada pelo protozoário Hepatozoon canis (H. canis), que acomete principalmente os carnívoros domésticos (Alencar et al., 1997). A transmissão para os cães ocorre após a ingestão de carrapatos contendo oocistos maduros de $H$. canis. Assume-se que Rhipicephalus sanguineus e Amblyomma spp sejam os principais vetores da doença em cães na América do Sul (Vicent-Johnson et al., 1997; O'Dwyer e Massard, 2001). Nos cães, a doença geralmente é intercorrente a outras enfermidades imunossupressoras (O'Dwyer e Massard, 2001), o que dificulta a individualização dos seus sinais clínicos. Craig (1998) assinala que os sinais mais freqüentes são anorexia, palidez de mucosas, emagrecimento e dores musculares.

O diagnóstico de rotina da enfermidade em cães baseia-se na identificação de células leucocitárias parasitadas, em esfregaços sangüíneos. Estudos recentes têm preconizado o uso da imunofluorescência indireta, do western blot e de biópsia tecidual como técnicas alternativas no diagnóstico (O’Dwyer e Massard, 2001).

Várias drogas são usadas no tratamento da hepatozoonose canina, embora com resultados controversos. O dipropionato de imidocarb tem apresentado resultados inconsistentes quando prescrito isoladamente. Entretanto, quando associado à tetraciclina ou à doxiciclina tem mostrado resultados satisfatórios (O'Dwyer e Massard, 2001). Macintire et al. (1997) atestaram a efetividade da sulfonamida/trimetoprim combinada com a pirimetamina, apesar de observarem recidiva da doença meses após o término da terapia.

No Brasil, Alencar et al. (1997) descreveram a infecção por $H$. canis em um "cão-do-mato" (Cerdocyon thous), ao destacarem a ocorrência em animais silvestres. Gondim et al. (1998) relataram a infecção natural em cães domésticos com sinais de anorexia, palidez de mucosas, febre, vômito, diarréia e dores musculares. Em virtude do escasso número de estudos nacionais envolvendo a casuística da hepatozoonose canina, o presente estudo objetivou relatar três casos de infecção canina por H. canis, ressaltando os principais achados clínicoepidemiológicos da doença.

Três cães infectados por $H$. canis foram encaminhados para o serviço ambulatorial de enfermidades infecciosas dos animais da Faculdade de Medicina Veterinária e Zootecnia UNESP/Botucatu-SP, entre maio de 1999 e janeiro de 2001. O diagnóstico de hepatozoonose foi firmado a partir da observação do parasita em esfregaços de sangue corados pelo método de Giemsa (Fig. 1).

Recebido para publicação em 5 de junho de 2003

E-mail: danmoura@usp.br 


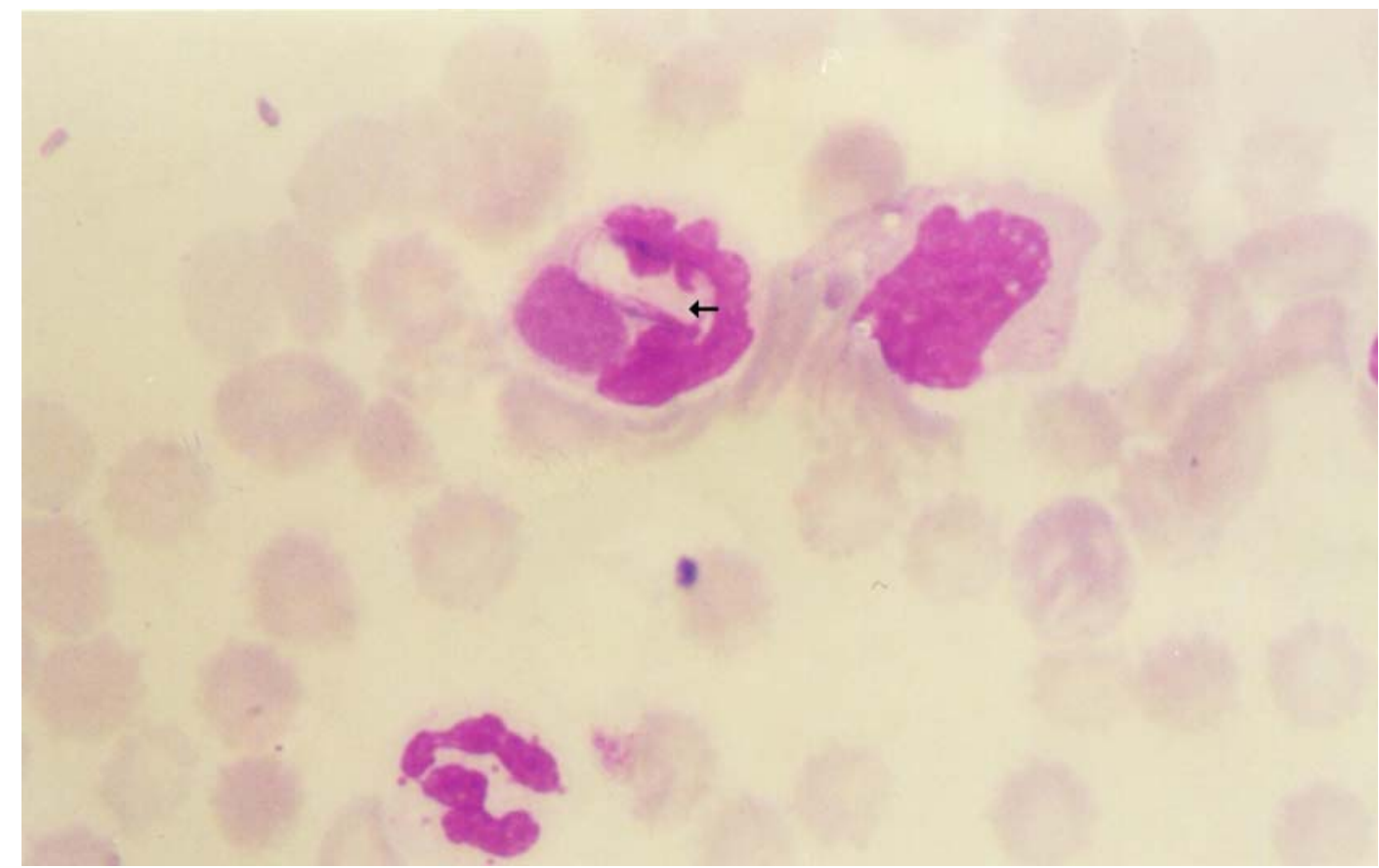

Figura 1. Fotomicrografia de Hepatozoon canis em neutrófilo (Giemsa, 1000X).

Exames hematológicos revelaram anemia regenerativa, leucocitose por neutrofilia, linfopenia e monocitose. Nos exames bioquímicos, a hiperglobulinemia ocorreu no animal sem raça definida e no da raça Pastor Alemão, que também apresentou hipoalbuminemia, aumento da gama glutamiltransferase (gama GT) e creatina-quinase (CK).

O cão da raça Fila (macho, três meses de idade, único procedente de área urbana) foi tratado com dipropionato de imidocarb $(5 \mathrm{mg} / \mathrm{kg}$, SID, IM, dose única) e tetraciclina $(22 \mathrm{mg} / \mathrm{kg}, \mathrm{BID}, \mathrm{VO}$, por 14 dias). O cão sem raça definida (macho, 18 meses de idade) e da raça Pastor Alemão (fêmea, nove anos de idade) receberam a associação de dipropionato de imidocarb $(5 \mathrm{mg} / \mathrm{kg}$, SID, IM, dose única) e sulfametoxazol/trimetoprim $(50 \mathrm{mg} / \mathrm{kg}$, SID, VO, por 15 dias). Todos os animais receberam reposição hidro-eletrolítica e energética, e complexos vitamínicos. Dos três cães tratados apenas o Fila morreu. Somente no animal sem raça definida foi constatada a presença de ectoparasitas.

De maneira similar ao presente relato, mesmo com o advento de novas técnicas para a identificação de $H$. canis, a grande maioria dos estudos fundamenta o diagnóstico na visualização do protozoário em células leucocitárias a partir de esfregaços de sangue (O’Dwyer e Massard, 2001). Macintire et al. (1997) observaram que a ocorrência da hepatozoonose não foi influenciada pelo sexo, raça e faixa etária dos animais. O cão sem raça definida apresentou febre, tosse, secreção óculonasal purulenta e linfadenopatia; o da raça Pastor-Alemão apresentou apatia, prostação, tremores musculares e dificuldade em manter-se em estação. Esses sintomas são considerados sugestivos de infecção por $H$. canis (VincentJohnson et al., 1997; Craig, 1998; O'Dwyer e Massard, 2001). Entretanto, a co-infecção de $H$. canis com doenças como parvovirose, cinomose, ehrliquiose e babesiose canina, dificultam sobremaneira a caracterização clínicolaboratorial da hepatozoonose canina (Craig, 1998; O'Dwyer e Massard, 2001). O cão da raça Fila apresentou diarréia com sangue, anorexia e vômito.

A diminuição nos níveis de albumina no Pastor Alemão e a elevação da globulina sérica no sem raça definida e no Pastor Alemão também foram relatadas em estudos similares (Vincent-Johnson et al., 1997; Craig, 1998; O’Dwyer e Massard, 
2001), e podem ser atribuídas, respectivamente, à queda na síntese protéica e à estimulação da resposta humoral induzida pela infecção por $H$. canis. No Pastor Alemão foi constatada elevação dos níveis da gama GT, justificada pelo desenvolvimento de hepatite secundária à agressão tissular provocada pelo agente (O’Dwyer e Massard, 2001), e elevação sérica de CK, decorrente de inflamação gerada pela multiplicação do $H$. canis na musculatura (Craig, 1998).

Os dados do presente relato alertam para a escassez de dados da ocorrência de hepatozoonose canina no país, para a complexidade dos achados clínico-laboratoriais em cães infectados por $H$. canis, assim como para a similaridade dos sintomas com outras doenças infecciosas em animais de companhia. Ressaltam, assim, a necessidade de investigações clínico-epidemiológicas conduzidas no intuito de fornecer subsídios para melhor caracterização da hepatozoonose canina no Brasil.

Palavras-chave: cão, Hepatozoon canis, epidemiologia, sinais clínicos

\begin{abstract}
Clinic-epidemiological data in canine infection by Hepatozoon canis were reported. Apatia, anorexia, prostaction, weight loss and muscle pain were the most-common clinical recognized signs. Blood smear was used as diagnosis method for the detection of $\mathrm{H}$. canis gamonts in the neutrophils. Anaemia, leucocytosis, monocytosis and lymphopenia were observed in the haematological exams. Imidocarb dipropionate associated with tetracyclines and sulfamethoxasole-trimethoprim were used in therapy.
\end{abstract}

Keywords: dog, Hepatozoon canis, epidemiology, clinical signs

\section{REFERÊNCIAS BIBLIOGRÁFICAS}

ALENCAR, N.X.; KOHAYAGAWA, A.; SANTARÉM, V.A. Hepatozoon canis infections of wild carnivores in Brazil. Vet. Parasitol., v.70, p.279-282, 1997.

BANETH, G.; AROCH, I.; PRESENTEY, B. Hepatoozon canis infection in a litter of Dalmatian dogs. Vet. Parasitol., v.70, p.201-206, 1997.

CRAIG, T.M. Hepatozoonosis. In: GREENE, C.E. Infectious diseases of the dog and cat. 2.ed. Philadelphia: WB Saunders, 1998. p.458-465.

GONDIM, L.F.; KOHAYAGAWA, A.; ALENCAR, N.X. et al. Hepatozoonosis in Brazil
: description of eigth naturally occurring cases. Vet. Parasitol., v.74, p.319-323, 1998.

MACINTIRE, D.K.; VICENT-JOHNSON, N.; DILLON, A.R. et al Hepatozoonosis in dogs : 22 cases (1989-1994). J. Am. Vet. Med. Assoc., v.210, p.916-922, 1997.

O'DWYER, L.H.; MASSARD, C.L. Aspectos gerais da hepatozoonose canina. Clin. Vet., v.31, p.34-40, 2001.

VICENT-JOHNSON, N.; MACINTIRE, D.K.; BANETH, G. Canine hepatozoonosis: pathophysiology, diagnosis, and treatment. Comp. Cont. Educ. Pract. Vet., v.19, p.51-65, 1997. 